\title{
The Effectiveness of Hands-on Health Informatics Skills Exercises in the Multidisciplinary Smart Home Healthcare and Health Informatics Training Laboratories
}

\author{
A. H. Sapci ${ }^{1}$ H. A. Sapci \\ ${ }^{1}$ Department of Allied Health, Adelphi University, Garden City, \\ New York, United States \\ 2 Hancock, Michigan, United States \\ Appl Clin Inform 2017;8:1184-1196.
}

\author{
Address for correspondence A. H. Sapci, MD, Department of Allied \\ Health, Adelphi University, Nexus Building Room 317, \\ 1 South Avenue, Garden City, NY 11530, United States \\ (e-mail: sapci@adelphi.edu).
}

\section{Abstract}

Keywords

- hands-on health informatics training

- smart home-based health platform

- remote patient monitoring

- experiential health informatics
Objective This article aimed to evaluate the effectiveness of newly established innovative smart home healthcare and health informatics laboratories, and a novel laboratory course that focuses on experiential health informatics training, and determine students' self-confidence to operate wireless home health monitoring devices before and after the hands-on laboratory course.

Materials and Methods Two web-based pretraining and posttraining questionnaires were sent to 64 students who received hands-on training with wireless remote patient monitoring devices in smart home healthcare and health informatics laboratories.

Results All 64 students completed the pretraining survey (100\% response rate), and 49 students completed the posttraining survey ( $76 \%$ response rate). The quantitative data analysis showed that $95 \%$ of students had an interest in taking more hands-on laboratory courses. Sixty-seven percent of students had no prior experience with medical image, physiological data acquisition, storage, and transmission protocols. After the hands-on training session, $75.51 \%$ of students expressed improved confidence about training patients to measure blood pressure monitor using wireless devices. Ninety percent of students preferred to use a similar experiential approach in their future learning experience. Additionally, the qualitative data analysis demonstrated that students were expecting to have more courses with hands-on exercises and integration of technology-enabled delivery and patient monitoring concepts into the curriculum.

Conclusion This study demonstrated that the multidisciplinary smart home healthcare and health informatics training laboratories and the hands-on exercises improved students' technology adoption rates and their self-confidence in using wireless patient monitoring devices. received

August 13, 2017

accepted after revision

November 8, 2017
DOI https://doi.org/ 10.4338/ACI-2017-08-RA0136.

ISSN 1869-0327. 


\section{Background and Significance}

Around the 1990s, before the Internet era, health informatics was only an academic discipline that required further consideration. ${ }^{1}$ Over the last decade, informatics tools and solutions evolved exponentially; consequently, these developments changed perspectives of patient management protocols, and increased biomedical knowledge generated new clinical practice and research areas. ${ }^{2}$

Telemedicine; remote patient monitoring; smart home for healthcare; data-driven medicine; and Predictive, Preventive, Personalized, Psycho-cognitive, and Participatory (P5) medicine have one common denominator: they all focus on data. Data-driven health informatics systems can identify and predict risk factors using new analytical approaches, and improve the quality of care at all health system levels. Existing information systems have the ability to use advanced data mining techniques and analytic algorithms. Since there is a growing market demand to develop new approaches to discovery, tomorrow's workforce needs specific skill sets to work with new data structures, interact with health data, and capture and store index data from medical devices and sensors.

\section{Remote Patient Monitoring and Smart Home-Based Health Platforms}

Smart home-based health platforms and remote patient monitoring technologies require specialized training along with specific infrastructure and equipment. The majority of nursing and health informatics students do not have access to robotic telepresence systems, infrared motion sensors, fall sensors, cordless monitor bed alarms, sleep tracking systems, and interactive web-based patient monitoring applications, and furthermore most of the training courses provide only theoretical foundations and do not include any hands-on component for patient monitoring training.

The age structure of the world's population has been changing in an unprecedented manner and according to the United Nation's report, population aged 60 or over projected to reach 2.1 billion in 2050, and 3.1 billion in $2100 .^{3}$ Rapidly aging population and increasing life expectancy requires new chronic disease prevention and management strategies to improve health outcomes and quality of life. The convergence of intelligent-connected technologies, advances in sensor and home-based health- as well as patient-monitoring devices, and the evolution of smart homes provided the clinicians the ability to develop new disease management methods and approaches using technology-based systems. Advances in wireless, wearable devices, and digital health technologies enable aging in place. Pilot studies have shown the benefits of patient monitoring in certain chronic conditions such as heart failure, and improved clinical outcomes. ${ }^{4}$ Smart homes equipped with new networked technologies such as motion detectors, pressure sensors, and smart appliances can enable automation; monitor physiological, mental, and social wellbeing; detect sleep problems, wandering, agitation, and behavioral changes; and help with the early diagnosis of various conditions such as dementia. ${ }^{5}$
Recent studies that focus on context-aware change detection models ${ }^{6}$ and care robots for independent living at home $e^{7}$ have shown promising results to detect specific chronic diseases in advance and increase the quality of life for older adults. The term Internet of Things (IoT) encompasses all network-enabled applications and devices that can communicate, interact, and transfer data, and this technology has the potential to collect vital data using wearable devices and sensors. ${ }^{8}$ IoT-based smart healthcare systems and devices have already become commercially available for individuals and smart homes. A recent systematic review determined a trend toward using remote patient monitoring technologies that consist of multiple components such as wearables, biosensors, computerized systems, and smartphones to assess and manage chronic conditions in the elderly. ${ }^{9}$

\section{The Need for Specialized Training}

The changing nature of medical practice and technological advances in care delivery requires innovative training models. Information extraction, data analytics, and specific stateof-the-art device usage skills have become crucial for both health informatics professionals and clinicians. Teaching core informatics, data science, and analytics skills using traditional training models and standard information technology infrastructure is quite challenging, and researchers have been proposing new models to keep education programs up to date with the changing landscape of informatics. ${ }^{10}$ Health informatics training for innovation has also recently gained much importance.

Graduate health informatics education generally focuses on the incorporation of core information and computational science skills such as information retrieval, knowledge representation, ontology, vocabulary, networking, statistics, data structures, and modeling into the curriculum. Hersh et al demonstrated the success of distance learning programs in health informatics over a decade ago ${ }^{11}$ and today there are no question marks about the educational value of online programs. Even though Commission on Accreditation for Health Informatics and Information Management Education (CAHIIM) and International Medical Informatics Association (IMIA) developed curriculum requirements for health informatics programs, $^{12}$ the methods to teach hands-on skills are still underdeveloped and vague.

It is quite challenging to gain technical skills from a health informatics curriculum that relies only on traditional lectures and reading the material. The TIGER Initiative representing more than 100 nursing leaders' collective vision supports hands-on electronic health record (EHR) training for nursing students. ${ }^{13}$ Although some health informatics programs have recently started adopting educational EHR training tools for the master of health informatics students, EHR education constitutes only a small part of health informatics curriculum. ${ }^{14}$

\section{The Need for Hands-on Training}

The Office of the National Coordinator (ONC) for Health Information Technology launched Workforce Development Program in 2009, and awarded four initiatives that train different staff for various roles. Community College Consortia to Educate Health 
InformationTechnology Professionals, Program of Assistance for University-Based Training, Curriculum Development Centers, and Competency Examination Programs initiatives developed the original Workforce Curriculum Development training materials. $^{15,16}$ A program evaluation conducted in 2013 determined that students were consistently requesting additional opportunities for hands-on experience. ${ }^{17}$ Seven grantees were awarded in 2015 to update the training materials, and subsequently, they added five new components. ${ }^{18}$

ONC for Health Information Technology Workforce Development Program ${ }^{17}$ recommends hands-on experience. Despite the fact that these components provide exposure to Department of Veterans Affairs' popular EHR system VistA, ${ }^{16}$ they do not include any training material about telemedicine and remote patient monitoring that will enhance clinicians' ability to monitor and treat patients in home care settings. A recent formative evaluation of the Workforce Development Program determined students', employers,' and instructors' wish for hands-on training and real-life experience and the need for new training strategies. ${ }^{19}$ Many medical education programs primarily focus on the effective use of EHRs, and do not provide necessary hands-on skills to conduct remote medical consultations and use store-and-forward technologies. ${ }^{20}$ The Association of American Medical Colleges (AAMC) and Howard Hughes Medical Institute (HHMI) defined scientific competencies for medical school graduates and recommended training in health informatics competencies to use and develop new models of clinical practice. $^{21}$

\section{Core Informatics Knowledge and Skills for Tomorrow's Health Informaticians and Clinicians}

Gardner et al described the core content for the subspecialty of clinical informatics, and determined mastery informatics domains as medical knowledge, mastery of fundamental information systems concepts, process re-engineering, system evaluation, and leadership skills. ${ }^{22}$ Safran et al defined program requirements for fellowship education in clinical informatics, ${ }^{23}$ and determined educational activities that provide skills to interpret and process the information as an information technology user or health and clinical informatics specialist. Existing competencies mainly focus on (1) core knowledge and skills; (2) health and biosciences, medicine, health system organization; and (3) informatics/computer science, mathematics, and biometry knowledge. ${ }^{24}$ However, there is still little focus on experiential training and technical competencies for innovation framework.

Today's medical specialties and subspecialties have unique technological needs. Many clinical and health informatics products in the market were developed with "one-size-fitsall" approach, and clinicians are actively looking for innovative ways to fulfill their specialties' custom needs especially in remote patient monitoring and home-care field. However, it is important to emphasize the difference between medical device invention and innovative use of systems and devices. Biomedical engineers collaborate with clinicians and scientists to design and invent medical devices. ${ }^{25}$ This process requires a sophisticated patent application and Food and Drug Administration's approval. On the other hand, clinician-innovators focus on designing solutions that will lead to transformations in health care, and this requires a nontraditional training. ${ }^{26}$

Innovation is an accepted strategy to build competitiveness. ${ }^{25}$ Recent developments in high-level programming languages, open source initiatives, the availability of medical development toolsets to the end user, growing remote patient monitoring, point-of-care device market, and the advances in interoperability standards allow skilled teams to innovate and develop their own applications and systems with a relatively low budget. Clinicians who work in academic medical centers have opportunities to collaborate with clinical informatics departments, and develop custom solutions if there is an institutional support for homegrown applications or systems. Loftus et al described a competition to motivate entrepreneurial teams to design and develop innovative solutions. ${ }^{27-29}$ Clinician-innovators are able to develop innovative solutions or applications with the help of health informaticians who have required educational background and hands-on skills to integrate various components and development kits that exist in the market. Moreover, health informaticians with a strong programming background can use their skills to develop new applications, add new features to existing open source applications, and also develop new models for university-industry collaborations. ${ }^{30}$

The speed of adoption of new health information systems generated a gap in informatics training. A recent study emphasizes the need for technology innovation training programs. ${ }^{25}$ Another study evaluated U.S.-based biomedical informatics training and identified minimal coverage areas in graduate curricula. ${ }^{31}$ It is important to integrate hands-on health informatics device and application training into the curriculum to provide core applied experiential informatics skills.

\section{Materials and Methods}

A novel laboratory course about remote patient monitoring, and clinical decision making with sensor data, was developed. It consists of a specific smart home for healthcare laboratory; a health informatics training laboratory; interactive on-ground and online lectures; and hands-on exercises to capture, mine, analyze, and visualize data. The main purpose of this training course is to bridge the gap in health informatics skills training.

\section{Laboratory Course Design}

Prior to developing the laboratory course, IMIA task force's core knowledge and skill lists were compared, ${ }^{32}$ and the similarities and differences between Certified Professional in Healthcare Information Management Systems (CPHIMS) and IMIA working group competencies were reviewed. ${ }^{24}$ Furthermore, IMIA's recommendations on health informatics and biomedical education $^{14}$ were analyzed, and the gaps in specific practical technical skill development were determined.

The laboratory course was designed to provide both practical and technical skills to monitor chronic disease patients at home, and delivered at the smart home and health informatics laboratories. It was composed of two parts. During the introductory part, all students received a lecture about the theoretical 
fundamentals of smart home and remote patient monitoring applications. After this part, students were divided into groups, and each group was given same hands-on exercises to learn how to perform real-time health monitoring with wireless glucomonitoring and blood-pressure monitoring systems. Then, they were asked to set up a personal health record account. Every participant paired their smartphone to a Bluetooth wireless blood pressure monitor and wireless gluco-monitoring systems, uploaded their data to the designated personal health record account, evaluated the strengths and weaknesses of wireless patient monitoring applications, and prepared a technology assessment report. In this study, technical skills to use patient monitoring devices and sensor pairing were selected for nursing students because they will be responsible for training chronic disease patients in the proper use of home-monitoring equipment.

\section{Smart Home for Healthcare and Health Informatics Training Laboratories}

The selection of interactive home telehealth, telemonitoring, and digital imaging equipment for the laboratories was determined after careful review and consideration of the American Telemedicine Association's Clinical Practice Guidelines. $^{33}$ The blueprints of the smart home and health informatics training laboratories, which are located at Adelphi University, New York, were designed by the first author. The smart home for home healthcare laboratory is the first part of the training environment and encompasses a state-ofthe-art videoconferencing equipment, two VGo robotic telepresence systems with accessories and handheld cameras, Riester ri-screen multifunctional digital camera systems with the general imaging and otoscopic lenses, AMD-3300 12-lead electrocardiogram (ECG) gloves for at-home monitoring with analytical software, Agnes interactive webbased collaborative patient assessment software to exchange medical documents and capture diagnostic device data, 40" HDTV, Amazon Echo that serves as a smart home automation hub, remote thermometers and monitoring systems, infrared monitor sensors, cordless monitor-bed alarms, caregiver pagers with motion sensor, sleep tracking systems with sleep sensors, cordless chair sensor pads, waterproof medical alert systems, motion sensors for central monitoring units, wireless alarms with bed and chair pads, central monitoring units, iHealth Sense wireless wrist blood pressure monitors, iHealth Feel wireless blood pressure monitors, iHealth Ease blood pressure monitors, iHealth Smart wireless gluco-monitoring systems, iHealth Align portable glucometers, iHealth Core wireless body composition scales, iHealth Air wireless pulse oximeters, Life Labs wireless remote thermometer and body temperature monitoring system, cordless, wireless alarm with bed and chair pads, Lifesource UA-851THX wireless auto blood pressure monitor, Sevenhugs hugOne sleep tracking system and two sleep sensors, medical alert systems for seniors, Smart Caregiver economy central monitoring unit and motion sensor, Smart Caregiver fall guard cordless monitor, Smart Caregiver GCT-WI cordless chair sensor pad with transmitter, Secure Wireless caregiver pager and PIR motion sensor for patient wandering and fall prevention, infrared monitor sensor for TL-2100G Cordless Monitor, Samsung Galaxy Tab S2 9.7", and Apple iPad Air 2 tablets. - Fig. 1 summarizes the conceptual design of smart home training laboratory. The second part of the training environment is the health informatics training laboratory, which is also equipped with interactive web-based collaborative patient assessment software (-Fig. 2).

The laboratories are designed to be used in multiple classes across all health informatics and nursing courses, and various remote patient monitoring devices transfer data from the smart home laboratory to the adjacent health informatics training laboratory. These state-of-the-art environments will give students the opportunity to engage in hands-on learning with three high-fidelity manikins and smart technologies. Health informatics students with information technology background will be able to extract and analyze sensor and medical device data, troubleshoot common sensor and wireless monitoring device problems, and learn how to understand patterns from data. And students with clinical background will be able to focus on clinical skills training, and they will learn how to operate and train patients, and make decisions using patient monitoring devices ( - Fig. 3 ).

\section{Survey Design}

A mixed design web-based survey that consists of both qualitative and quantitative questions was developed, and sent to students via the academic e-mail link. All students were given 1 week to complete the questionnaire. The results were kept confidential, and two reminders were sent after the course. Likert scales (1-5) with anchors ranging from 1 (Not likely at all or Strongly disagree) to 5 (Extremely likely or Strongly agree) depending on the question type were used to measure students' feedback. In addition, open-ended questions to provide further comments about students' experience and suggestions were used. - Table 1 lists the questions used in this research.

\section{Questionnaire Validation}

Face validity was established by subject-matter experts. To ensure congruity between items and domain, two expert faculty members reviewed scale items. They were provided with a verbal overview in the laboratories, and the conceptual framework and practical considerations were discussed. The experts provided feedback during all phases, evaluated the clarity and consistency, and suggested alternative wording when necessary. Sixteen master's degree students were asked to complete the questionnaire to evaluate the clarity of wording. Subsequently, the questionnaire was refined, and modifications were made based on participants' feedback.

Cronbach's $\alpha$ was used to calculate the instrument quality and internal consistency. For the overall reliability, the Cronbach's $\alpha$ value was $0.919(N=63)$. This result indicated a satisfactory internal consistency and construct validity.

\section{Study Subjects}

Sixty-four students out of possible 103 from the fourth-year cohort of undergraduate nursing students were recruited for this study. The inclusion criteria for students' participation were: (1) their informed consent and (2) currently enrolled 
Wireless Health Data Exchange Simulations

\section{Smart Home Lab}
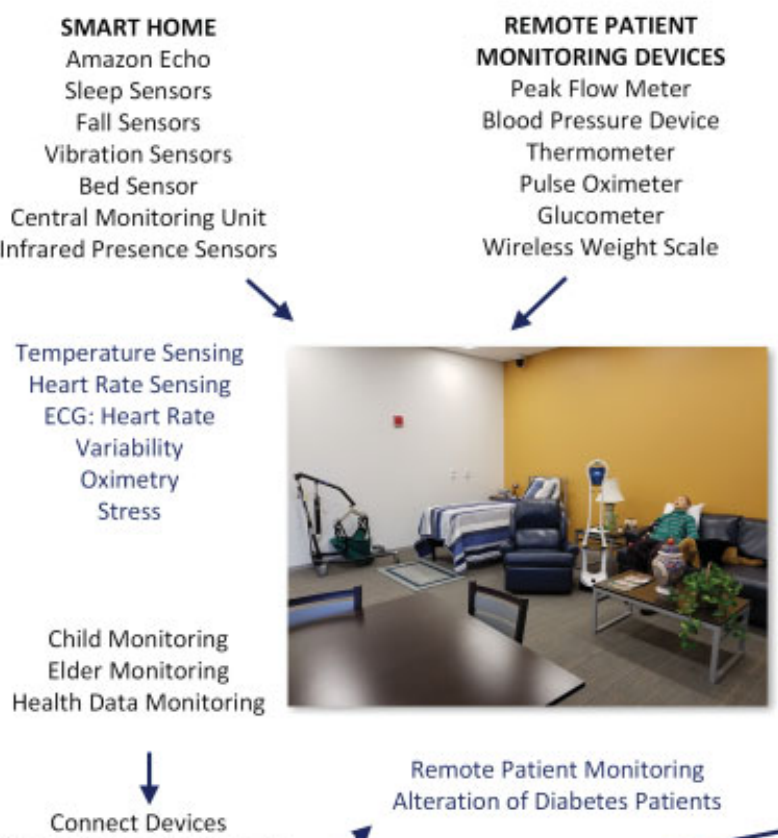

(Connectivity, Intelligence)

Collect Data

(Sensors, Storage)
Remote Patient Monitoring Alteration of Diabetes Patients

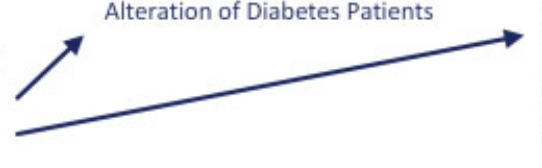

Health Informatics Lab
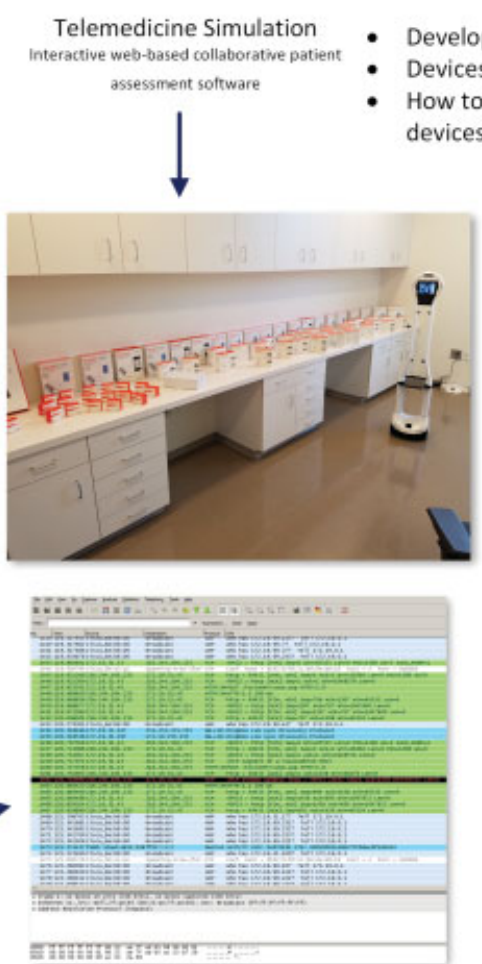

- Development applications for students

- Devices collect isolated data

- How to collect data from different devices?

Fig. 1 Smart home and health informatics laboratories: conceptual design.

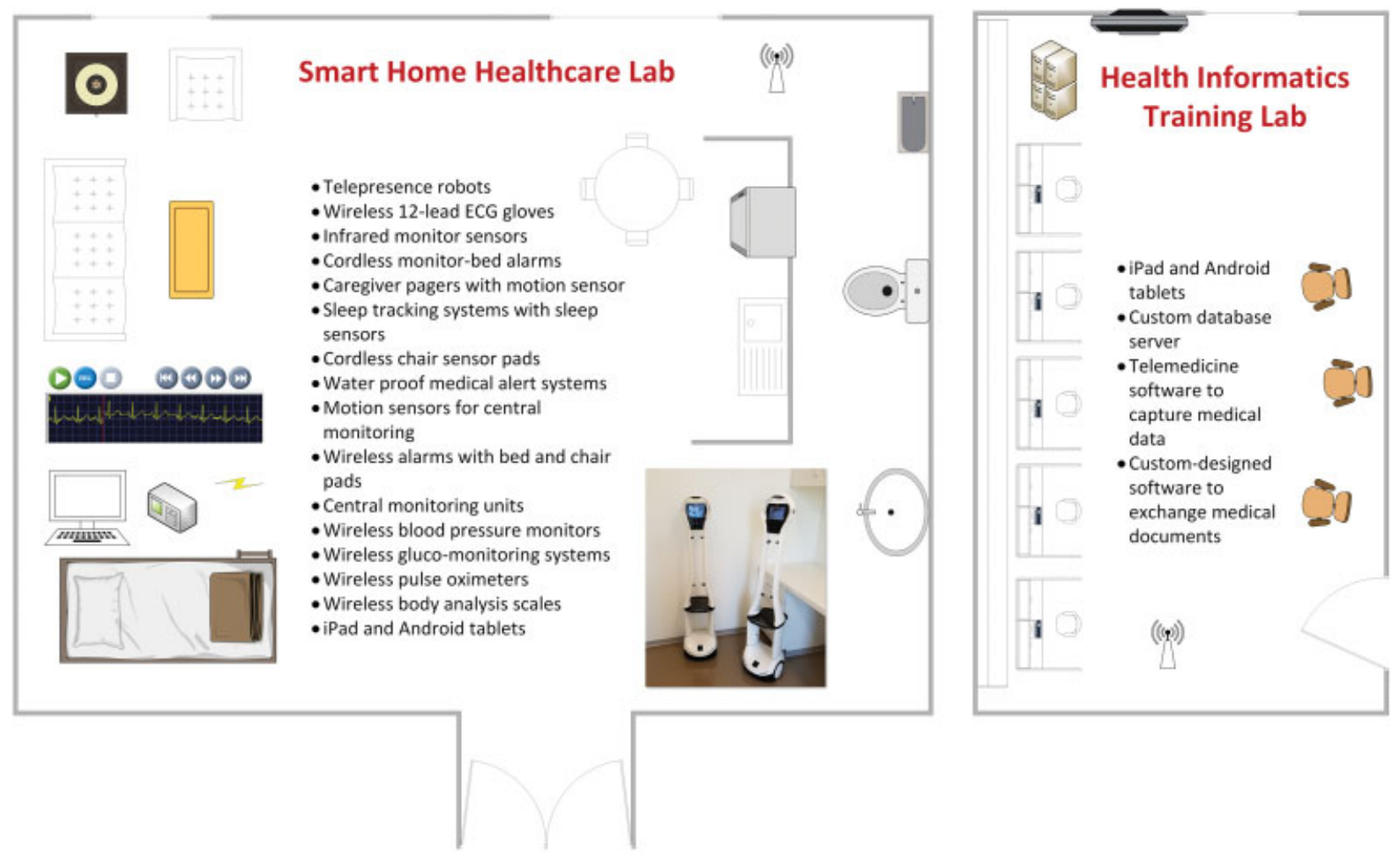

Fig. 2 Smart home and health informatics laboratories: floor plan. 


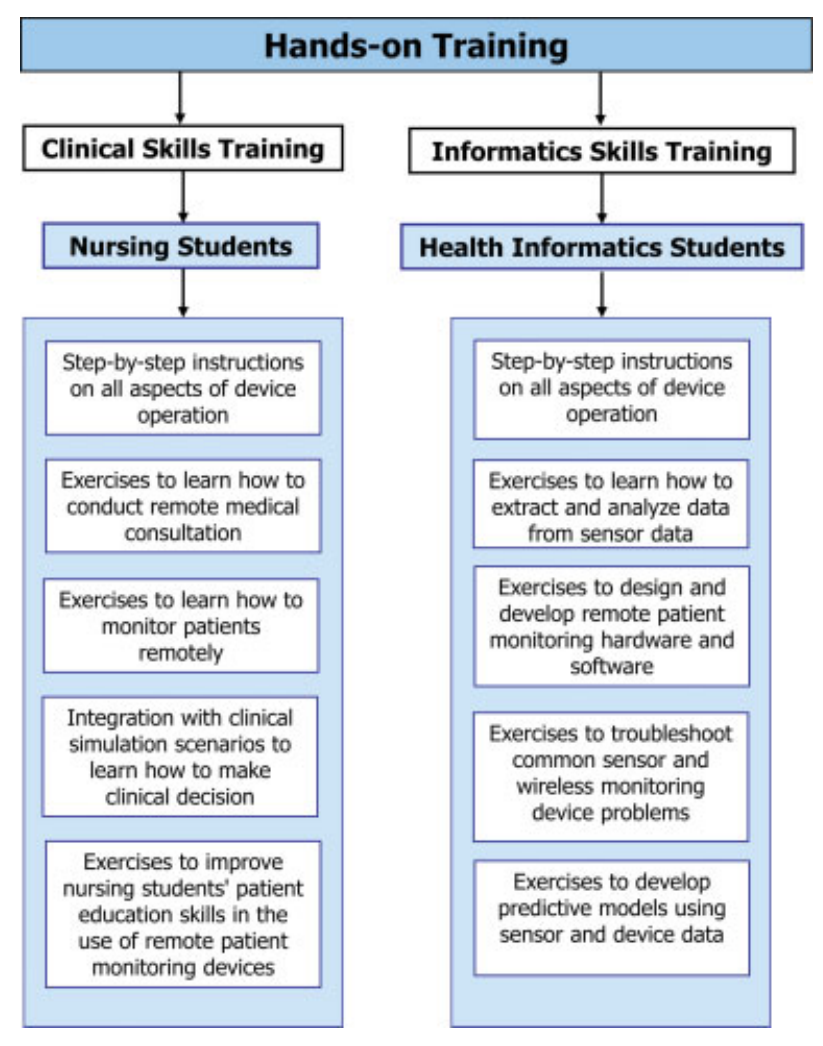

Fig. 3 Hands-on skills training. in the Community Health Nursing course. All participating students were enrolled at the beginning of the Spring 2017 semester.

\section{Data Collection}

All participants were asked to complete the web-based questionnaire. Additionally, there was a consent form with detailed information about the study on the first page of the survey. There was no compensation or extra credit, and the participation was voluntary. Participants received an invitation to complete the questionnaire 1 week before the scheduled training day. After the training, participants received another invitation. Completing the questionnaire was considered as students' consent to participate. Students were informed about the purpose of the study.

\section{Measurement Tools}

The study data were entered into Microsoft Excel, and statistical analyses were performed using IBM Statistical Package for the Social Sciences (SPSS) 23.0. To examine differences in relation to pre- and posttraining confidence in using wireless monitoring devices, Mann-Whitney $U$-test and Wilcoxon's signed-rank tests were selected. Qualitative data gathered in the questionnaires were analyzed with text analysis software NVivo 11. Open-ended questions were coded into a thematic framework reflecting participants' perceptions.

Table 1 Questionnaire design

\begin{tabular}{|c|c|}
\hline Quantitative research & Pretraining questions \\
\hline & $\begin{array}{l}\text { - I am familiar with telemedicine and remote patient monitoring device characteristics and have } \\
\text { necessary knowledge and skills to teach my patients how to use them } \\
\text { - Rate your confidence in using wireless monitoring devices } \\
\text { - Would you like to have experiential learning opportunities about new health informatics tools, } \\
\text { applications, and devices? } \\
\text { - I am familiar with medical image and physiological data acquisition, storage, retrieval, and } \\
\text { transmission protocols } \\
\text { - Our current curriculum provides necessary knowledge and skill to use the latest medical devices } \\
\text { and applications. } \\
\text { - Our current curriculum provides the necessary knowledge to analyze healthcare data and skills } \\
\text { - I conduct data-based reasoning using the latest patient monitoring technologies. } \\
\text { Posttraining Questions } \\
\text { - I feel that I now have enough knowledge to teach my patients how to track and share vital blood } \\
\text { pressure data using wireless patient monitoring devices } \\
\text { - After the hands-on training, rate your confidence in using wireless monitoring devices } \\
\text { - Do you think that teaching how to use remote patient monitoring devices and telemedicine } \\
\text { devices should be a priority for our curriculum? } \\
\text { - I prefer to learn in such approach in my future learning } \\
\text { - After the hands-on training, I will likely recommend using wireless monitoring devices to my } \\
\text { patients }\end{array}$ \\
\hline \multirow{2}{*}{$\begin{array}{l}\text { Qualitative research } \\
\text { questions }\end{array}$} & Open-ended question (posttraining) \\
\hline & $\begin{array}{l}\text { - Do you have any new requests or ideas for new courses, certification programs, practical } \\
\text { exercises that will focus on new technologies, wireless patient monitoring, telemedicine, and } \\
\text { health informatics applications? }\end{array}$ \\
\hline
\end{tabular}




\section{Results}

Sixty-four nursing students, of which 13 were males and 51 were females, participated in this study. All students completed the pretraining survey, yielding a response rate of $100 \%$, and 49 students completed the posttraining survey, representing a response rate of $76 \%$.

\section{Quantitative Data Analysis}

For the quantitative analysis, a Likert scale of five points was used to measure student responses ( $1=$ Strongly disagree and 5 = Strongly agree). The majority of the students provided positive feedback regarding the hands-on experience (-Fig.4):
$75.51 \%$ of respondents (37/49) "strongly agreed" or "agreed" felt that they had enough knowledge to teach their patients how to track and share vital blood pressure data using wireless patient monitoring device after the training session.

Sixty-seven percent of students stated that they were not familiar with medical image, physiological data acquisition, storage, and transmission protocols ( - Table 2). Twenty-five percent of students agreed or strongly agreed, and 23\% of students neither agreed nor disagreed with the statement which reads "Our current curriculum provides necessary knowledge and skills to use the latest medical devices and applications." Only 22\% of students disagreed or strongly disagreed with this statement (-Table 2). Thirty-one percent

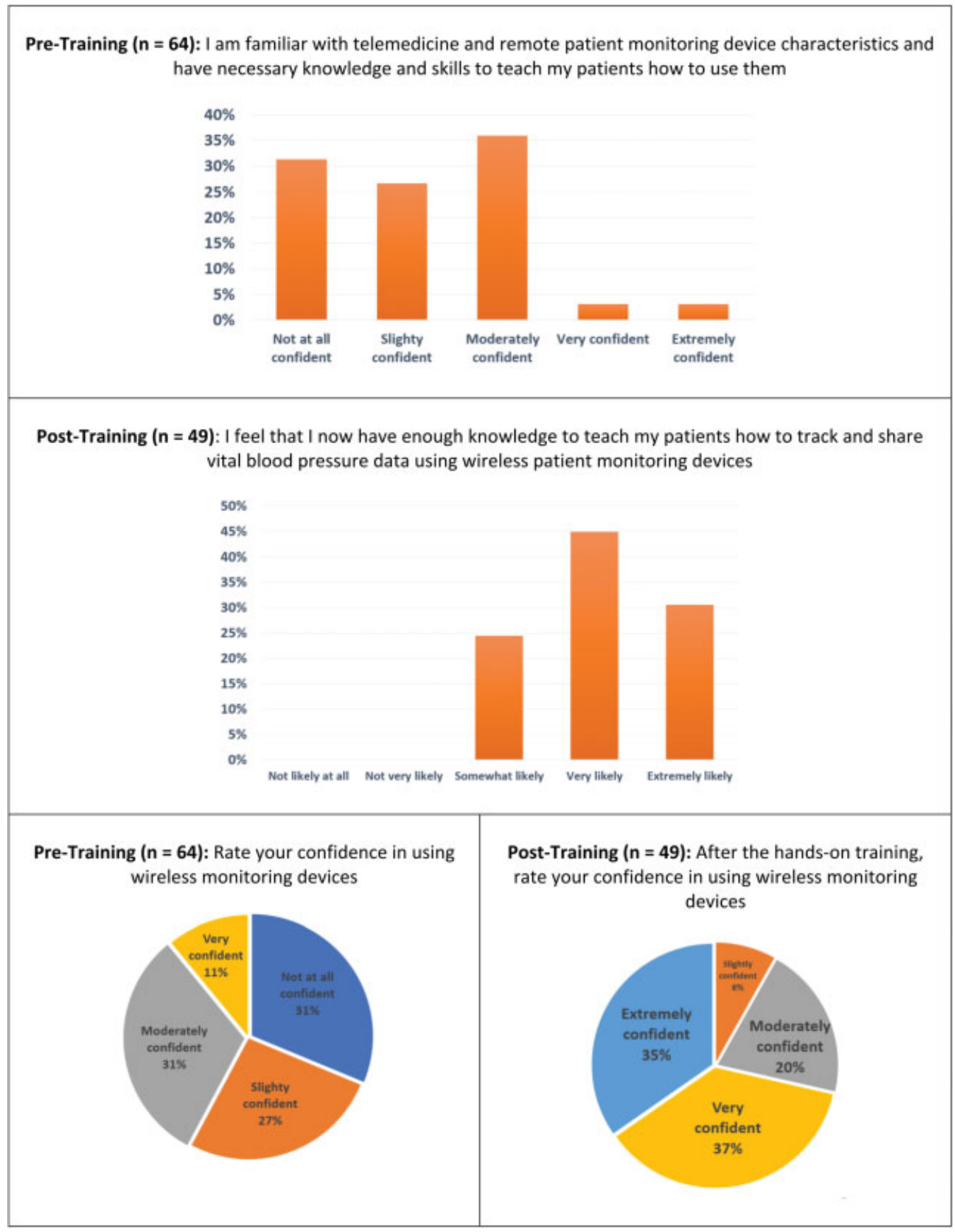

Fig. 4 Pre- and posttraining evaluations. 
Table 2 Students' perceptions

\begin{tabular}{|c|c|c|c|c|c|c|c|c|c|c|}
\hline \multicolumn{11}{|l|}{ Pretraining students' perceptions } \\
\hline & \multicolumn{2}{|c|}{$\begin{array}{l}\text { Not at all } \\
\text { confident }\end{array}$} & \multicolumn{2}{|c|}{$\begin{array}{l}\text { Slightly } \\
\text { confident }\end{array}$} & \multicolumn{2}{|c|}{$\begin{array}{l}\text { Moderately } \\
\text { confident }\end{array}$} & \multicolumn{2}{|c|}{$\begin{array}{l}\text { Very } \\
\text { confident }\end{array}$} & \multicolumn{2}{|c|}{$\begin{array}{l}\text { Extremely } \\
\text { confident }\end{array}$} \\
\hline & \multicolumn{2}{|c|}{$n=64 \%$} & \multicolumn{2}{|c|}{$n=64 \%$} & \multicolumn{2}{|c|}{$n=64 \%$} & \multicolumn{2}{|c|}{$n=64 \%$} & \multicolumn{2}{|c|}{$n=64 \%$} \\
\hline $\begin{array}{l}\text { I am familiar with medical image } \\
\text { and physiological data acquisition, } \\
\text { storage, retrieval, and } \\
\text { transmission protocols }\end{array}$ & 22 & 34 & 21 & 33 & 17 & 27 & 3 & 5 & 1 & 2 \\
\hline \multicolumn{11}{|l|}{ Pretraining students' perceptions } \\
\hline & \multicolumn{2}{|c|}{$\begin{array}{l}\text { Strongly } \\
\text { disagree }\end{array}$} & \multicolumn{2}{|c|}{ Disagree } & \multicolumn{2}{|c|}{$\begin{array}{l}\text { Neither } \\
\text { agree or } \\
\text { disagree }\end{array}$} & \multicolumn{2}{|c|}{ Agree } & \multicolumn{2}{|c|}{$\begin{array}{l}\text { Strongly } \\
\text { agree }\end{array}$} \\
\hline & \multicolumn{2}{|c|}{$n=64 \%$} & \multicolumn{2}{|c|}{$n=64 \%$} & \multicolumn{2}{|c|}{$n=64 \%$} & \multicolumn{2}{|c|}{$n=64 \%$} & \multicolumn{2}{|c|}{$n=64 \%$} \\
\hline $\begin{array}{l}\text { Our current curriculum provides } \\
\text { necessary knowledge and skill } \\
\text { to use the latest medical } \\
\text { devices and applications }\end{array}$ & 5 & 8 & 9 & 14 & 34 & 23 & 15 & 23 & 1 & 2 \\
\hline $\begin{array}{l}\text { Our current curriculum provides } \\
\text { the necessary knowledge } \\
\text { to analyze healthcare data } \\
\text { and skills to conduct data-based } \\
\text { reasoning using the latest } \\
\text { patient monitoring technologies }\end{array}$ & 1 & 2 & 7 & 11 & 36 & 56 & 20 & 31 & 0 & 0 \\
\hline
\end{tabular}

of respondents agreed with the statement "Our current curriculum provides the necessary knowledge to analyze healthcare data and skills to conduct data-based reasoning using the latest patient monitoring technologies" and 56\% were unsure, they neither agreed nor disagreed. Only $13 \%$ of students disagreed or strongly disagreed (-Table 2 ). The learning objective of the laboratory course did not include analytical skills; therefore, these three questions were only asked before the training to determine students' overall knowledge about home monitoring, telemedicine applications, and perceptions about the curriculum.

Both evidence-based protocols and U.S. National High Blood Pressure Education Program's Joint National Committee reports emphasize the role of nurses in patient education. ${ }^{34}$ To determine future nurse educators' ability to train patients, a question about nursing students' adoption of wireless patient monitoring was asked before and after the training session (-Table 3).

Table 3 Students' perceptions

\begin{tabular}{|c|c|c|c|c|c|c|c|c|c|c|}
\hline \multicolumn{11}{|l|}{ Pretraining students' perceptions } \\
\hline & \multicolumn{2}{|c|}{$\begin{array}{l}\text { Not } \\
\text { likely at } \\
\text { all }\end{array}$} & \multicolumn{2}{|c|}{$\begin{array}{l}\text { Not very } \\
\text { likely }\end{array}$} & \multicolumn{2}{|c|}{$\begin{array}{l}\text { Somewhat } \\
\text { likely }\end{array}$} & \multicolumn{2}{|c|}{ Very likely } & \multicolumn{2}{|c|}{$\begin{array}{l}\text { Extremely } \\
\text { likely }\end{array}$} \\
\hline & \multicolumn{2}{|c|}{$n=63 \%$} & \multicolumn{2}{|c|}{$n=63 \%$} & \multicolumn{2}{|c|}{$n=63 \%$} & \multicolumn{2}{|c|}{$n=63 \%$} & \multicolumn{2}{|c|}{$n=63 \%$} \\
\hline $\begin{array}{l}\text { I will likely recommend using } \\
\text { wireless monitoring } \\
\text { devices to my patients }\end{array}$ & 1 & 2 & 3 & 5 & 30 & 48 & 21 & 33 & 8 & 13 \\
\hline \multicolumn{11}{|l|}{ Posttraining students' perceptions } \\
\hline & \multicolumn{2}{|c|}{$\begin{array}{l}\text { Not } \\
\text { likely at } \\
\text { all }\end{array}$} & \multicolumn{2}{|c|}{$\begin{array}{l}\text { Not very } \\
\text { likely }\end{array}$} & \multicolumn{2}{|c|}{$\begin{array}{l}\text { Somewhat } \\
\text { likely }\end{array}$} & \multicolumn{2}{|c|}{ Very likely } & \multicolumn{2}{|c|}{$\begin{array}{l}\text { Extremely } \\
\text { likely }\end{array}$} \\
\hline & \multicolumn{2}{|c|}{$n=49 \%$} & \multicolumn{2}{|c|}{$n=49 \%$} & \multicolumn{2}{|c|}{$n=49 \%$} & \multicolumn{2}{|c|}{$n=49 \%$} & \multicolumn{2}{|c|}{$n=49 \%$} \\
\hline $\begin{array}{l}\text { After the hands-on training, I will } \\
\text { likely recommend using wireless } \\
\text { monitoring devices to my patients }\end{array}$ & 0 & 0 & 1 & 2 & 10 & 20 & 24 & 49 & 14 & 29 \\
\hline
\end{tabular}


Table 4 Confidence in using wireless monitoring devices

\begin{tabular}{|c|c|c|c|c|c|}
\hline & \multicolumn{2}{|c|}{$\begin{array}{l}\text { Pretraining group } \\
(n=64)\end{array}$} & \multicolumn{2}{|c|}{$\begin{array}{l}\text { Posttraining group } \\
(n=49)\end{array}$} & \multirow[t]{2}{*}{$p$-Value } \\
\hline & Mean & SD & Mean & SD & \\
\hline $\begin{array}{l}\text { Rate your confidence in using wireless } \\
\text { monitoring devices }\end{array}$ & 2.22 & 1.01 & 3.98 & 0.95 & 0.000 \\
\hline
\end{tabular}

Mann-Whitney $U$-test and Wilcoxon's tests were applied to examine differences between pre- and posttraining students' confidence in using wireless monitoring devices. Mann-Whitney $U$-test showed that there was a significant difference between the two groups ( $p \leq 0.01$ ). Wilcoxon's signed-rank test results indicated that there was a significant difference between the means of pre- and posttraining groups ( - Table 4 and -Fig. 4). Respondents reported a significant improvement in perceived self-confidence to operate wireless home health monitoring devices. The overall score for the pre- and posttraining questionnaire was $2.22 \pm 1.01$ versus $3.98 \pm 0.95(p<0.000)$.

After the training session, $95 \%$ of students expressed an interest in taking more courses that contain similar hands-on exercises about new health informatics tools, applications, and devices. Ninety percent of students stated that they would prefer this learning approach that focuses on handson skills and competencies with state-of-the-art wireless devices in an experiential learning environment. Similarly, $49 \%$ of students selected essential and high priority options, and $43 \%$ selected medium priority option for the "Do you think that teaching how to use remote monitoring and telemedicine device should be a priority for our curriculum?" question. Only $4 \%$ thought this was not a priority (Low priority or Not a priority) (-Fig. 5).

\section{Qualitative Data Analysis}

A thematic analysis in accordance with Braun and Clarke's analytical framework was performed to identify patterns. ${ }^{35}$ From the dataset, initial codes were generated, and three major themes were identified. All students were asked one open-ended question about their requests or ideas for new courses and practical exercises that will focus on new technologies, wireless patient monitoring, and health informatics application, and 29 students responded.

\section{Theme 1: Students' Expectations of Hands-on Health Informatics Skills}

According to the students' narratives, they expressed the need for more resources and courses that include hands-on exercises (-Table 5).

Theme 2: Students' Expectations Related to Specific Home Care and Remote Patient Monitoring Training Participants extensively expressed their expectations to have courses that include ECG monitors, remote fetal monitoring, fall sensors, wireless, and Global Positioning System (GPS)enabled patient monitoring devices (-Table 5).
Theme 3: Students' Expectations Related to TechnologyEnabled Delivery Methods and Technology Integration into the Curriculum

Participants emphasized the need to integrate informatics concepts that contain remote patient monitoring applications into the nursing curriculum ( - Table 5 ).

\section{Study Limitations}

This study has limitations. The sample size was relatively small, and students' previous experience with technology was not examined. In addition, the study provides only an evaluation of one particular feature of the smart home and health informatics laboratories, and therefore the results cannot be generalized to other settings.

This research included nursing students only; however, the laboratory course framework can be applied to larger and diverse health informatics students.

\section{Discussion and Conclusion}

The growth of the population aged 60 years or over around the globe led to a greater demand for the treatment of noncommunicable diseases associated with old age, and increased the need for personal care. ${ }^{36}$ Smart home technologies can play a vital role in increasing patients' independence and comfort. ${ }^{37}$ Health information technology training is an essential part of chronic disease management. After a thorough needs assessment process, a laboratory course was developed to implement a structured hands-on technical telehealth and remote patient monitoring skills. Health informatics and clinical research informatics applications require specialized equipment, and new training models to capture and analyze data. Ammenwerth et al recommended including site visits to medical centers in the curriculum to learn more about hospital information systems. ${ }^{38}$ Although this is still a good recommendation, visit alone cannot provide enough practical experience. Students who will work in informatics fields should have hands-on problem-solving skills with tools to support practice and research.

Schools of medicine and allied health sciences have been shifting toward problem-based learning curriculum over the last four decades. ${ }^{39}$ Problem-based learning focuses on realworld challenges, and has become a widely accepted teaching strategy for clinical practice scenarios. ${ }^{40}$ An innovative health informatics curriculum should provide an opportunity for experiential learning, and demonstrate a significant correlation among the visualization of clinical data for real-time diagnosis, continuous monitoring, and the ability to understand evolving 


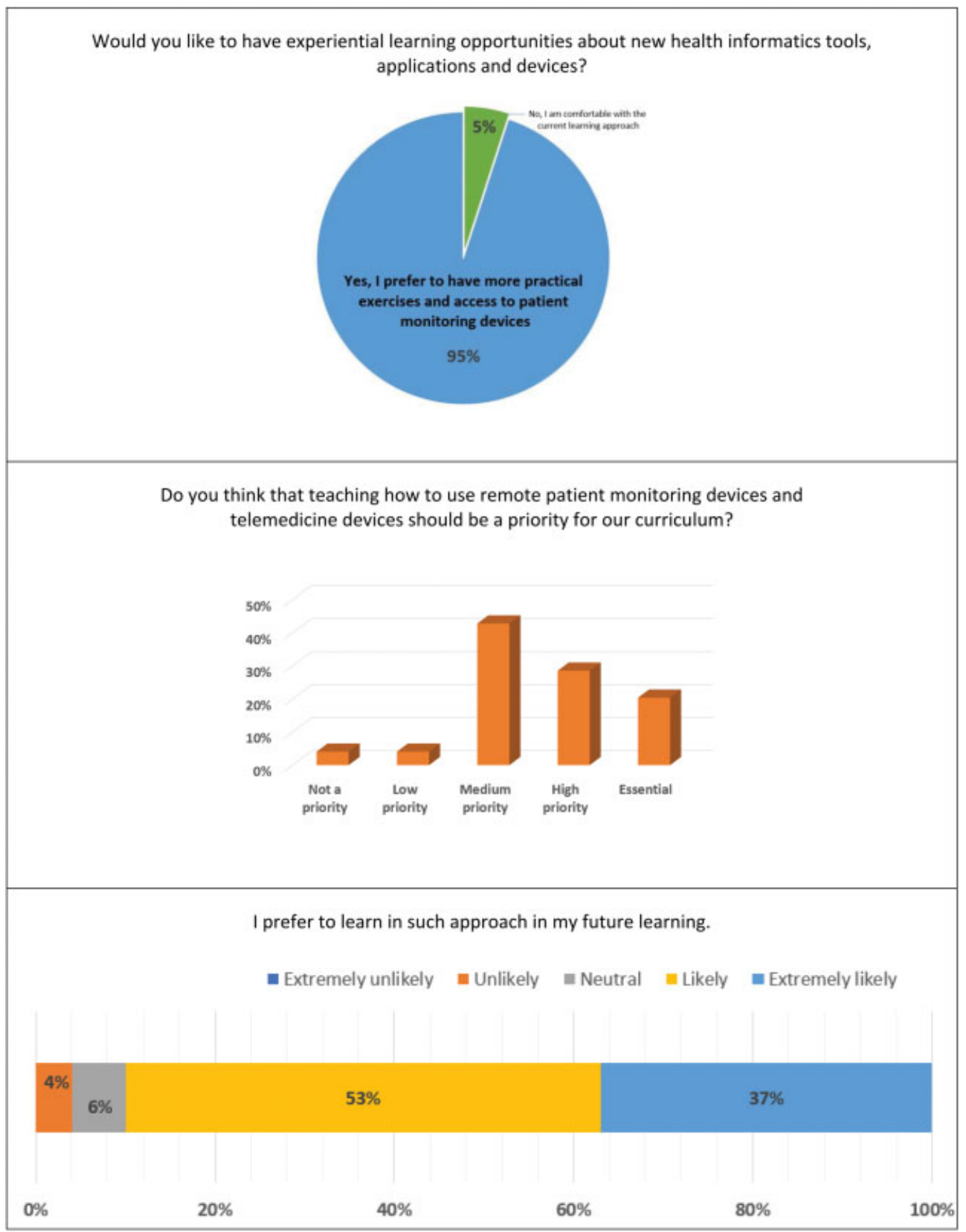

Fig. 5 Pre- and posttraining evaluations.

concepts of health informatics. Traditional didactic lecturebased learning focuses on the passive transfer of knowledge, and has limited effect on problem solving. ${ }^{41,42}$ ONC health information technology curriculum includes some sections about hands-on laboratory courses, but those courses mostly address EHR training; there is little focus on innovation, remote patient monitoring education, and experiential training. ${ }^{43}$

This article described the infrastructure of two health informatics skills training laboratories, and demonstrated students' strong interest to participate in hands-on learning that teaches remote patient monitoring and smart home applications. With the existing technology in these laboratories, students can conduct simulated telemedicine visits, transfer vital signs, extract data from these devices and sensors, save them to the local computer, write and compile custom codes with existing tools, have opportunities to learn how to operate the devices, extract data from USB and Bluetooth connections, mine and analyze the data, and integrate these tools to support medical practice with reallife-like scenarios. Such practical exercises will provide an opportunity to gain real-life experience with wireless monitoring systems; teach the integration of these devices with home monitoring technologies; understand how to capture data; learn how to integrate various medical devices, hardware and software, to build new systems depending on clinicians' needs; and evaluate students' feedbacks. 
Theme 1: Students' expectations of hands-on health informatics skills

"More hands-on and resources should be available to student to be comfortable in the practical setting."

"As we are moving more towards technology-based health care settings, hands-on practice for students is a good experience."

"With the rise of telemedicine, I believe everyone should know how to use Bluetooth settings. Even in the demo there were problems with trying to connect the device. I think every student should be learning how to use technology moving forward."

"I still feel that we should have more hands-on experience in the curriculum to assist us in real-world situations."

"Portable ECG monitors; some of us have never seen a portable ECG monitor, and the wireless monitoring devices.

Maybe adding some to community clinical could make the community experience rich. We read these things in book, or watched them on ATI, but seeing, and touching, and using/practicing with them will enhance knowledge in teaching clients how they work."

"I wish that I will become very confident in using the latest medical devices and applications."

Theme 2: Students' expectations related to specific home care and remote patient monitoring technology training

"It would be good if we had a course that includes:

- How to use fall sensors and GPS systems for patients at risk for falls and wandering

- How to use cardiac monitoring devices such as EKG monitors

- How to use devices that connect HCPs to other HCPs (e.g., some areas have devices that will connect a neurologist to an ER that does not have to quickly diagnose stroke victims and get them faster and better care)"

"Portable medication administration devices."

"Prenatal fetal monitoring."

"I would like the equipment to be utilized for psychiatric patients to either improve clinical practice or to use it as a clinical makeup day, how to use fall sensors, medication adherence applications, portable ECG monitors, and wireless monitoring devices, lifting devices, and the pump."

"A course for the wireless blood pressure machine will be beneficial to the students as well as the patients. Once you get set up and know how to properly utilize the portable machines, it saves a lot of time and can help patients monitor and keep track of their health."

"Students would benefit from using the hands-on practice during the home visit Sim-Lab."

Theme 3: Students' expectations related to technology-enabled delivery methods and technology integration into the curriculum

"I wish we could integrate new and innovative ideas that we will be seeing in our future healthcare careers so that we can be up to date with the technology. Being knowledgeable now will help us educate our patients in the future." "I would like to see technology implemented into our curriculum."

"I think that the use of remote patient monitoring should be included in the learning curriculum. This way, students not only get to read about it but also see it in practice. By allowing students this opportunity, it will surely enhance our learning experience and prepare us for the vast products in telemedicine."

"The curriculum does not integrate technology in our classes. I think there should be a class just dedicated to technology that would be used most often in the clinical setting."

"Courses or certificate programs should be provided, either added into our curriculum or provided as a program/certification.

Practice should be provided with new technologies." "The day and age we live in always has new upcoming electronic technology that we should be prepared to use."

"It would be interesting to develop an entire patient assessment with data obtained purely through telehealth technology, and then formulate nursing diagnoses from these data. The robot device may assist in this area as well."

There is an increasing need for innovative theoretical and practical curricula, and future studies will concentrate on health informatics students' hands-on learning experience to extract data, write new applications, and develop new prototype systems using smart devices such as Amazon Alexa, fall sensors, bed alarms, and infrared motion sensors. Practical use of patient monitoring devices is not usually included in the graduate curricula because the access to state-of-the-art medical devices requires a specific infrastructure, investment, and trained clinicians and instructors.

One lesson learned in this study was the importance of laboratory staff's continuous support during the laboratory course. Highly interactive hands-on exercises require more than one skilled laboratory staff to provide support during the class. Another lesson learned was that interference from other wireless devices caused slower performance during certain times of the day, and it required IT department's help. Connecting to a 5-GHz wireless network and changing the course hours solved this problem. Because hard-wired outlets were designed for laboratory equipment only, the room did not have enough power outlets for students' laptops and mobile devices. Office of Facilities Management solved this problem by installing multiple electrical outlets and power strips.

This new replicable laboratory course framework to train next-generation health informaticians has the potential to serve as a model for further research. The dissemination of similar laboratories and focus on competencies will provide an opportunity to distinguish health informatics students' practical skills. 


\section{Multiple Choice Question}

Which of the following statement is wrong?

A. Office of the National Coordinator for Health Information Technology (ONC) Workforce Development Program recommends hands-on laboratory exercises

B. An evaluation of the Workforce Development Program determined students', employers', and instructors' desire for hands-on training

C. Workforce Development Program includes practical components about telemedicine and patient monitoring

D. The Association of American Medical Colleges (AAMC) and Howard Hughes Medical Institute (HHMI) defined recommended training in health informatics competencies for medical school graduates

E. TIGER Initiative supports hands-on training for nursing students

Correct Answer: The correct answer is C. The Workforce Development Program provides hands-on exposure to VistA EHR system, but does not include any practical components about telemedicine and patient monitoring.

\section{Protection of Human and Animal Subjects}

The study was performed in compliance with the World Medical Association Declaration of Helsinki on Ethical Principles for Medical Research Involving Human Subjects and was reviewed by Adelphi University's Institutional Review Board.

Conflict of Interest

None.

\section{References}

1 Greenes RA, Shortliffe EH. Medical informatics. An emerging academic discipline and institutional priority. JAMA 1990; 263(08):1114-1120

2 Cano I, Lluch-Ariet M, Gomez-Cabrero D, et al; Synergy-COPD Consortium. Biomedical research in a Digital Health Framework. J Transl Med 2014;12(Suppl 2):S10

3 World Population Prospects. The 2017 Revision, Key Findings and Advance Tables. United Nations, Department of Economic and Social Affairs, Population Division2017. Available at: https://esa. un.org/unpd/wpp/Publications/Files/WPP2017_KeyFindings.pdf

4 Kim KI, Gollamudi SS, Steinhubl S. Digital technology to enable aging in place. Exp Gerontol 2017;88:25-31

5 Chan M, Estève D, Escriba C, Campo E. A review of smart homespresent state and future challenges. Comput Methods Programs Biomed 2008;91(01):55-81

6 Forkan ARM, Khalil I, Tari Z, Foufou S, Bouras A. A context-aware approach for long-term behavioral change detection and abnormality prediction in ambient assisted living. Pattern Recognit 2015; 48(03):628-641

7 Fischinger D, Einramhof P, Papoutsakis K, et al. Hobbit, a care robot supporting independent living at home: first prototype and lessons learned. Robot Auton Syst 2016;75:60-78

8 Minerva R, Biru A, Rotondi D. Towards a definition of the Internet of Things (IoT). IEEE Internet Initiative; 2015. Available at: http:// iot.ieee.org/images/files/pdf/IEEE_IoT_Towards_Definition_Internet_of_Things_Revision1_27MAY15.pdf. Accessed July 15, 2017
9 Vegesna A, Tran M, Angelaccio M, Arcona S. Remote patient monitoring via non-invasive digital technologies: a systematic review. Telemed J E Health 2017;23(01):3-17

10 Friedman CP, Altman RB, Kohane IS, et al; American College of Medical Informatics. Training the next generation of informaticians: the impact of "BISTI" and bioinformatics-a report from the American College of Medical Informatics. J Am Med Inform Assoc 2004;11(03):167-172

11 Hersh W, Junium K, Mailhot M, Tidmarsh P. Implementation and evaluation of a distance learning introductory course in medical informatics. Stud Health Technol Inform 2001;84(Pt 2):1023-1027

12 Hasman A, Mantas J. IMIA accreditation of health informatics programs. Healthc Inform Res 2013;19(03):154-161

13 The TIGER Virtual Demonstration Collaborative Team. A TIGER Collaborative Report. 2009. Available at: http://s3.amazonaws. com/rdcms-himss/files/production/public/FileDownloads/tigerreport-virtual-learning-center.pdf. Accessed July 15, 2017

14 Milano CE, Hardman JA, Plesiu A, Rdesinski RE, Biagioli FE. Simulated electronic health record (Sim-EHR) curriculum: teaching EHR skills and use of the EHR for disease management and prevention. Acad Med 2014;89(03):399-403

15 The Office of the National Coordinator for Health Information Technology. Health IT Workforce Development Program. Available at: https://www.healthit.gov/sites/default/files/pdf/fact-sheets/getthe-facts-about-health-it-workforce-development-program. pdf. Accessed July 15, 2017

16 Mohan V, Abbott P, Acteson S, et al. Design and evaluation of the ONC health information technology curriculum. J Am Med Inform Assoc 2014;21(03):509-516

17 The Office of the National Coordinator for Health Information Technology, U.S. Department of Health and Human Services. Implementation of ONC's Workforce Development Program. 2013. Available at: https://www.healthit.gov/sites/default/files/communitycollegeevaluationsitevisitreport.pdf. Accessed July 15, 2017

18 The Office of the National Coordinator for Health Information Technology, U.S. Department of Health and Human Services. Workforce Training Program Awardees. Available at: https:// www.healthit.gov/providers-professionals/workforce-developmentprograms. Accessed July 15, 2017

19 NORC at the University of Chicago. Final Report: Executive Summary. Evaluation of the Information Technology Professionals in Health Care ("Workforce") Program - Summative Report. 2014. Available at: https://www.healthit.gov/sites/default/files/workforceevaluationsummativerpt_execsummary.pdf. Accessed July 15, 2017

20 Sikka N, Choudhri T, Jarrin R. The George Washington University Emergency Medicine Telemedicine and Digital Health Fellowship. Virtual Mentor 2014;16(12):976-980

21 Triola MM, Friedman E, Cimino C, Geyer EM, Wiederhorn J, Mainiero C. Health information technology and the medical school curriculum. Am J Manag Care 2010;16(12, Suppl HIT):SP54-SP56

22 Gardner RM, Overhage JM, Steen EB, et al; AMIA Board of Directors. Core content for the subspecialty of clinical informatics.J Am Med Inform Assoc 2009;16(02):153-157

23 Safran C, Shabot MM, Munger BS, et al; AMIA Board of Directors. Program requirements for fellowship education in the subspecialty of clinical informatics.J Am Med Inform Assoc 2009;16(02):158-166

24 Moore RA, Berner ES. Comparing health/medical informatics graduate program curricula against two sets of professional criteria. J Healthc Inf Manag 2004;18(03):44-50

25 Brinton TJ, Kurihara CQ, Camarillo DB, et al. Outcomes from a postgraduate biomedical technology innovation training program: the first 12 years of Stanford Biodesign. Ann Biomed Eng 2013;41(09):1803-1810

26 Zuckerman B, Margolis PA, Mate KS. Health services innovation: the time is now. JAMA 2013;309(11):1113-1114

27 Loftus PD, Elder CT, D’Ambrosio T, Langell JT. Addressing challenges of training a new generation of clinician-innovators through an interdisciplinary medical technology design program: 
bench-to-bedside. Clin Transl Med 2015;4(1). Available at: http:// www.clintransmed.com/content/4/1/15. Accessed July 15, 2017

28 Loftus PD, Elder CT, Sorensen MW, et al. Creating a Benchmark Medical Technology Entrepreneurship Competition. The University of Utah Bench-to-Bedside Medical Device Design Competition. NCIIA Open Conference Report; 2014. Available at: http:// venturewell.org/open2014/wp-content/uploads/2013/10/LANGELL.pdf. Accessed July 15, 2017

29 The University of Utah. The University of Utah Health Sciences Center for Medical Innovation: Bench-to-Bedside Competition Report 2012. 2012. Available at: https://library.med.utah.edu/ e-channel/wp-content/uploads/2016/06/EndofYearReport2012.pdf. Accessed July 15, 2017

30 Gelijns AC, Thier SO. Medical innovation and institutional interdependence: rethinking university-industry connections. JAMA 2002;287(01):72-77

31 Ritko AL, Odlum M. Gap analysis of biomedical informatics graduate education competencies. AMIA Annu Symp Proc 2013; 2013:1214-1223

32 Mantas J, Ammenwerth E, Demiris G, et al. Recommendations of the International Medical Informatics Association (IMIA) on education in biomedical and health informatics-first revision. Acta Inform Med 2010;18(01):4

33 American Telemedicine Association. Practice Guidelines \& Resources. Available at: http://hub.americantelemed.org/resources/telemedicine-practice-guidelines

34 Himmelfarb CRD, Commodore-Mensah Y, Hill MN. Expanding the role of nurses to improve hypertension care and control globally. Ann Glob Health 2016;82(02):243-253
35 Braun V, Clarke V. Using thematic analysis in psychology. Qual Res Psychol 2006;3(02):77-101

36 United Nations. World Population Ageing. 2015. Available at: http:// www.un.org/en/development/desa/population/publications/pdf/ ageing/WPA2015_Report.pdf

37 Demiris G, Rantz M, Aud M, et al. Older adults' attitudes towards and perceptions of "smart home" technologies: a pilot study. Med Inform Internet Med 2004;29(02):87-94

38 Ammenwerth E, Knaup P, Winter A, et al. On teaching international courses on health information systems. Lessons learned during 16 years of Frank - van Swieten Lectures on strategic information management in health information systems. Methods Inf Med 2017;56(Open):e39-e48

39 Sweeney G. The challenge for basic science education in problem-based medical curricula. Clin Invest Med 1999;22(01): $15-22$

40 Demiris G, Zierler B. Integrating problem-based learning in a nursing informatics curriculum. Nurse Educ Today 2010;30(02): 175-179

41 Zhao B, Potter DD. Comparison of lecture-based learning vs discussion-based learning in undergraduate medical students. J Surg Educ 2016;73(02):250-257

42 Choi E, Lindquist R, Song Y. Effects of problem-based learning vs. traditional lecture on Korean nursing students' critical thinking, problem-solving, and self-directed learning. Nurse Educ Today 2014;34(01):52-56

43 Miles MB, Huberman AM, Saldaña J. Qualitative data analysis: a methods sourcebook. 3rd ed. Thousand Oaks, CA: Sage Publications, Inc; 2014:381 